Case Report

\title{
Bilateral Intracavernous Carotid Artery Aneurysms Presenting as Diplopia in a Young Patient
}

\author{
Nikolaos Kopsachilis, ${ }^{1}$ Maria Pefkianaki, ${ }^{1}$ Gianluca Carifi, ${ }^{1}$ and Ioannis Lialias ${ }^{2}$ \\ ${ }^{1}$ Moorfields Eye Hospital, London EC1V 2PD, UK \\ ${ }^{2}$ Aristotle University of Thessaloniki, Thessaloniki, Greece
}

Correspondence should be addressed to Nikolaos Kopsachilis; nickkops@hotmail.com

Received 11 November 2012; Revised 26 January 2013; Accepted 17 February 2013

Academic Editor: Murat Ugurlucan

Copyright (C) 2013 Nikolaos Kopsachilis et al. This is an open access article distributed under the Creative Commons Attribution License, which permits unrestricted use, distribution, and reproduction in any medium, provided the original work is properly cited.

Introduction. Bilateral intracavernous carotid artery aneurysms (ICAAs) are extremely rare and difficult to treat. Case Report. A 26-year-old female presented in our clinic with acute diplopia due to oculomotor nerve palsy on the left side. Magnetic resonance imaging of the brain showed two heterogeneously enhanced masses indicating bilateral ICAA. An endovascular coil embolization was performed on the left side successfully, resulting in resolution of her symptoms. Conclusion. Thorough systemic evaluation in young patients with diplopia can reveal life-threatening underlying pathology and prevent major complications.

\section{Introduction}

Artery aneurysms are found in $5 \%$ of the general population and become symptomatic in $9 / 100.000$, most frequently in the fifth and sixth decades of life [1]. Intracavernous carotid artery aneurysms (ICAAs) represent less than $1 \%$ of intracranial aneurysms and show a slight female predominance [2]. Bilateral intracavernous carotid artery aneurysms are extremely rare and difficult to treat.

We report an unusual case of bilateral ICAA in a very young female patient presented as acute diplopia and treated with endovascular coil embolization.

\section{Case Report}

A 26-year-old woman was referred to our clinic with transient worsening diplopia. She also complained of headaches and had no further general motor or sensory symptoms. The patient had no history of hypertension or hypercholesterolemia and was not on any medications. Visual acuity was $6 / 5$ in the right eye and 6/6 in the left eye. Intraocular pressures were $13 \mathrm{~mm} \mathrm{Hg}$ both sides. Slit lamp examination was normal, and fundoscopy findings were unremarkable in both eyes. Orthoptic examination showed oculomotor nerve palsy on the left side. Other cranial nerves including abducens and trochlear nerves were intact. No systemic diseases such as Ehlers-Danlos, Paget's disease, or Marfan's syndrome were recorded.

With regard to the clinical findings, magnetic resonance imaging (MRI) of the brain was scheduled. Coronal and axial $\mathrm{T}_{1}$ weighted images demonstrated two heterogeneously enhanced masses indicating bilateral ICCA (Figure 1). Bilateral internal carotid arteries were visualized as flow voids encircling the mass.

An endovascular coil embolization using a Guglielmi detachable microcoil was performed on the left side uneventfully, in our Department of Neuroradiology. The patient tolerated the procedure well, and there were no major postoperative complications. A postoperative MRI scan showed successful thrombosis of the left aneurysm (Figure 2). One month after embolization, her diplopia had completely resolved. In view of the good postoperative result, an identical procedure on the right side was performed uneventfully 3 months later. At 6 months after initial presentation, the patient had recovered completely and had no recurrence of her diplopia. 


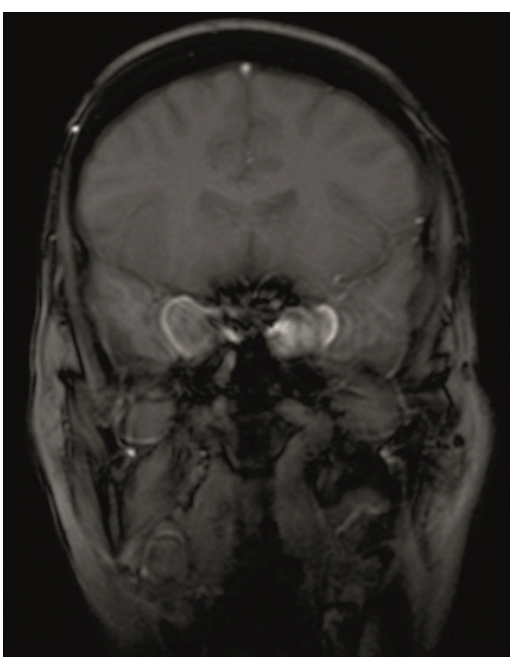

(a)

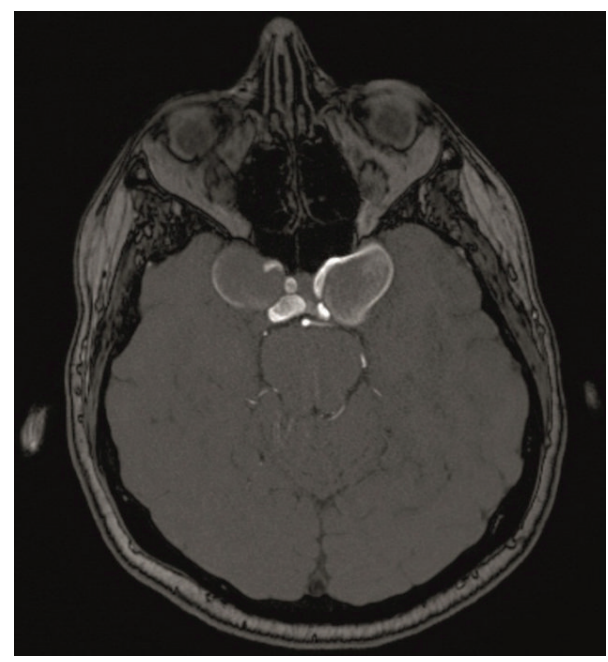

(b)

FIGURE 1: $\mathrm{T}_{1}$ weighted MRl of coronal (a) and (b) axial slices in a 26-year-old patient with transient diplopia and oculomotor nerve palsy on the left side. Two heterogeneously enhanced masses and an enhanced thin outer membrane can be recognized, indicating bilateral intracavernous carotid artery aneurysms.

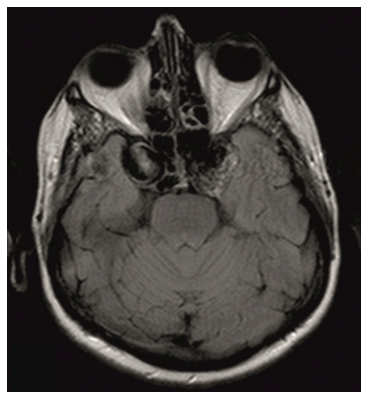

(a)

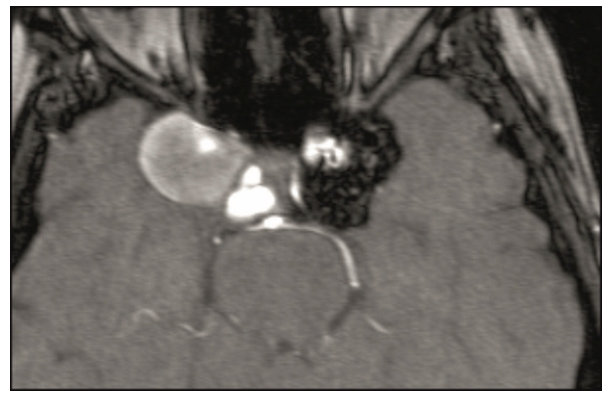

(b)

FIgURE 2: $\mathrm{T}_{1}$ weighted MRl axial slices of the same patient 2 days after treatment of the left intracavernous carotid artery aneurysm with endovascular coil embolization (a). Note the slight hypointensity due to thrombosis in the region of the previous left aneurysm (b).

\section{Discussion}

The etiology of giant aneurysms is multifactorial and many structural and hemodynamic stress factors have been previously discussed. However, pathogenesis of the cavernous aneurysm is not yet defined, and idiopathic intracavernous aneurysms are the most common [3].

Many bilateral intracavernous aneurysm cases have definite causative factors suggesting weakness of the carotid arterial wall. They can occur after radiation therapy or in association with connective tissue disorders such as fibromuscular dysplasia and Paget's Disease [4]. Furthermore, infectious (mycotic and bacterial) intracavernous aneurysms have been reported in the past [5].

The likelihood of an aneurysm becoming symptomatic is directly related to its size. The most common cause of symptoms (90\%) is rupture which can result in severe subarachnoid hemorrhage. Symptoms start with diplopia, photophobia nausea, and vomiting following respiratory arrest with a $50 \%$ death ratio. Rupture can also result in arteriovenous fistula or posterior fossa hematoma. In addition, stroke-like symptoms can be created by thrombosis. If the aneurysm is large enough, it compresses surrounding structures and causes progressive neuropathy. In this situation, the symptoms are progressive and less resulting in visual loss, diplopia, ptosis, and other focal deficits. The symptoms are directly related to the cranial nerves (III, IV, VI, VII, and VI) that cross the cavernous sinus [5].

Optimal management of symptomatic giant carotid aneurysms remains controversial. However, present treatment options favor bypass or embolization to direct surgery with very good results, as in our case [6].

To summarize we report an interesting case of bilateral ICAA in 26-year-old female presented as acute diplopia and emphasize the need of a thorough systemic evaluation in young patients with diplopia.

\section{Conflict of Interests}

The authors declare no conflict of interests. 


\section{References}

[1] V. A. Purvin, "Neuro-ophthalmic aspects of aneurysms," International Ophthalmology Clinics, vol. 49, no. 3, pp. 119-132, 2009.

[2] M. E. Linskey, L. N. Sekhar, W. Hirsch Jr., H. Yonas, and J. A. Horton, "Aneurysms of the intracavernous carotid artery: clinical presentation, radiographic features, and pathogenesis," Neurosurgery, vol. 26, no. 1, pp. 71-79, 1990.

[3] L. N. Sekhar, D. Ramanathan, D. K. Hallam, B. V. Ghodke, and L. J. Kim, "What is the correct approach to aneurysm management in 2011?" World Neurosurgery, vol. 75, no. 3-4, pp. 409-411, 2011.

[4] T. Rehman, R. Ali, C. Taylor, and H. Yonas, "Bilateral giant cavernous carotid artery aneurysms in a child with juvenile Paget's disease," World Neurosurgery, vol. 73, no. 6, pp. 691-693, 2010.

[5] B. R. Seo, T. S. Kim, S. P. Joo, and S. I. Jung, "Endovascular treatment of infective aneurysms of the bilateral cavernous sinus: case report and review of the literature," Clinical Neuroradiology, vol. 19, no. 2, pp. 162-165, 2009.

[6] J. M. Zabramski, R. F. Spetzler, and C. G. McDougall, "Percutaneous intracranial stent placement for aneurysms," Journal of Neurosurgery, vol. 99, no. 1, pp. 23-30, 2003. 


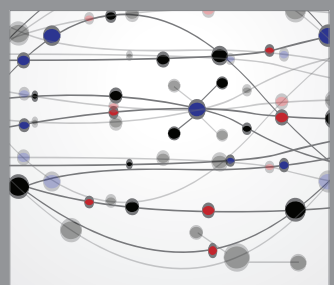

The Scientific World Journal
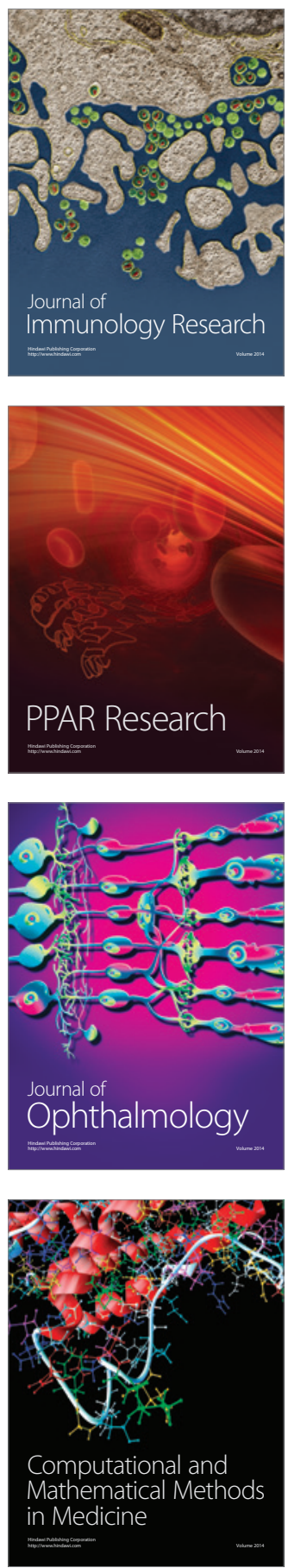

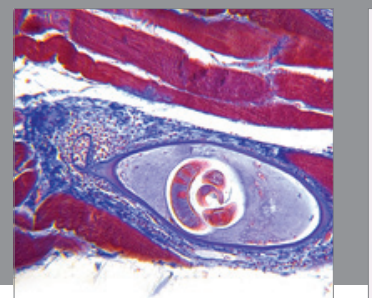

Gastroenterology

Research and Practice
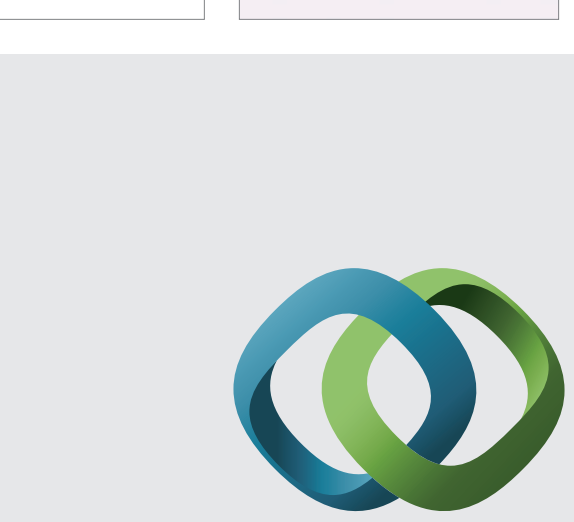

\section{Hindawi}

Submit your manuscripts at

http://www.hindawi.com
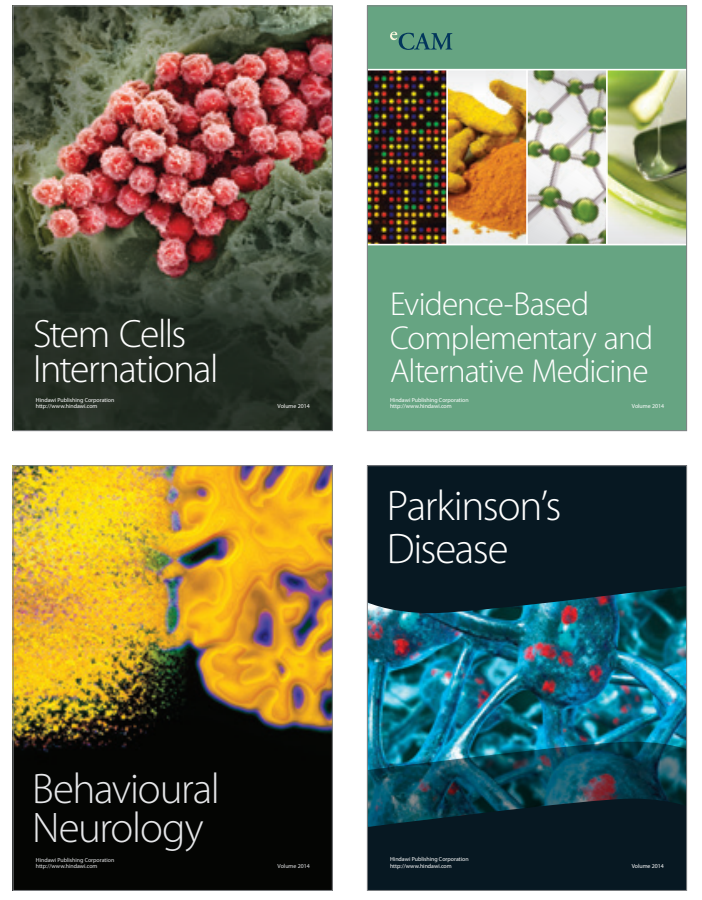
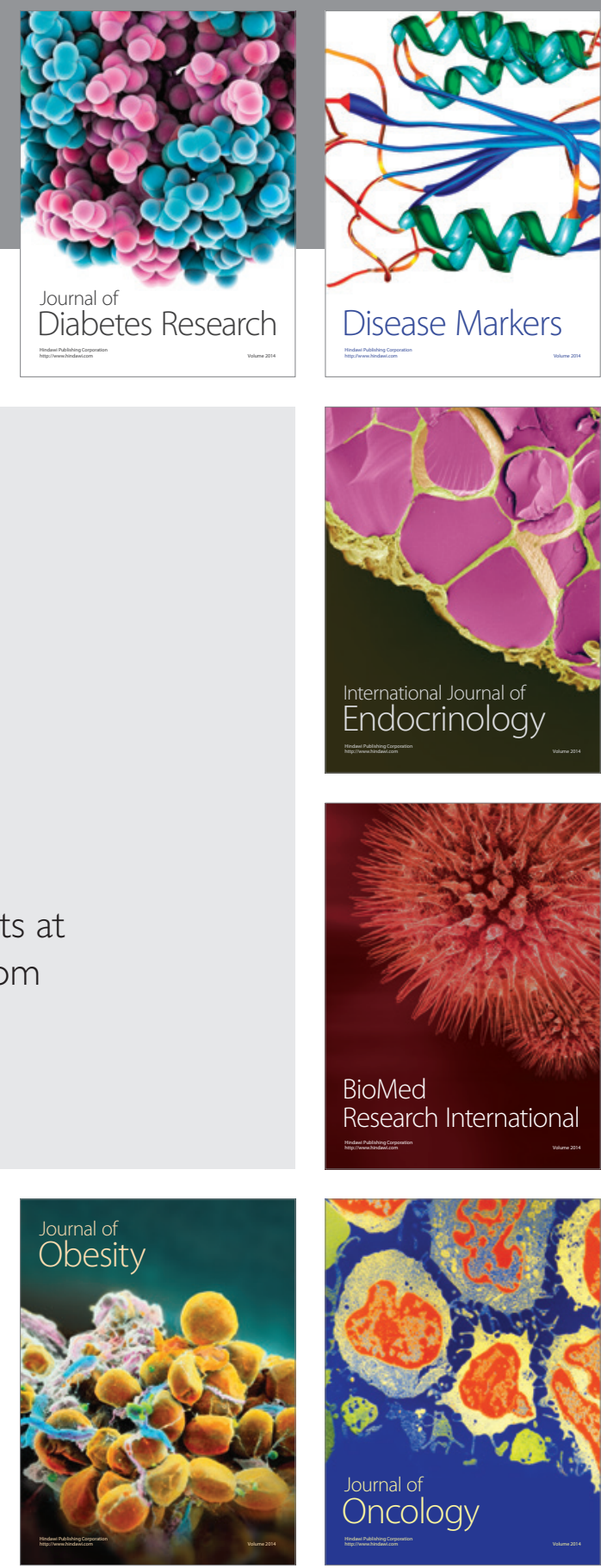

Disease Markers
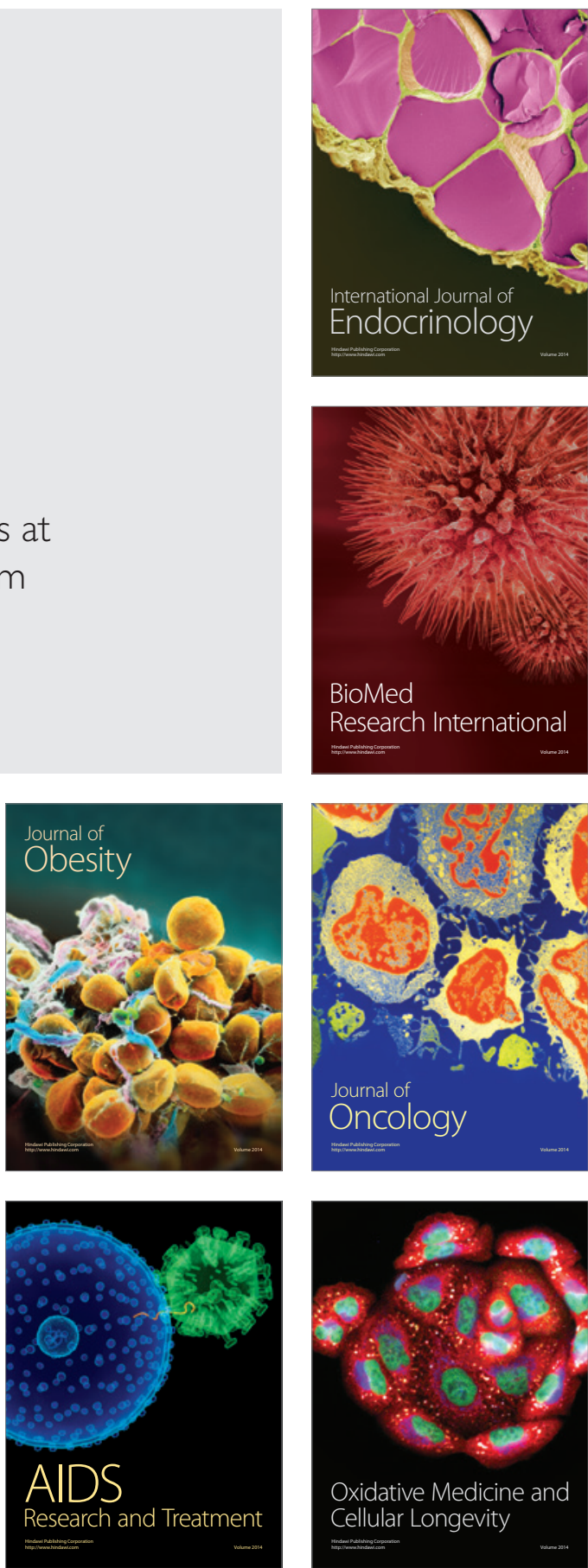